\title{
Microwave photonic network for active electronically scanned array radar
}

\author{
MANISHA MATHUR ${ }^{1}$, JAYNENDRA KUMAR RAI ${ }^{2}$ AND NILAKANTAN SRIDHAR
}

\begin{abstract}
Active electronically scanned array (AESA) radar has large number of transmit/Receive (T/R) modules which require multiple microwave and digital signals. Distribution of these signals through conventional method such as coaxial cable, twisted pair, etc. not only introduces engineering complexities and signal loss but also have limitation of bandwidth, data rate, transmission distance, etc. This paper addresses design and implementation of microwave photonic network for distribution of microwave and digital signals over single optical fiber using wavelength division multiplexing for AESA radars. The design challenge is to limit the variation in output radio frequency power within $\pm 1 \mathrm{~dB}$ over full operational band of radar from 2 to $4 \mathrm{GHz}$ and functionality under hostile military environment. Optical amplifiers have been used in all channels to stabilize optical output independent of wavelength with automatic light control. The optical signal is split into 64 identical parts to feed multiplexed signal into different digital receivers physically spread across the antenna array. It is an additional challenge to normalize performance as all 64 receivers show variation in output in spite of identical electronic circuitry. Experimental results validate the feasibility of microwave photonic network for wide branching distribution of multiple microwave and digital signals for AESA radar.
\end{abstract}

Keywords: Microwave photonics, Radar applications, Active phased array radar, Microwave photonic network, RF over fiber, Wavelength division multiplexing

Received 22 November 2015; Revised 4 February 2016; Accepted 8 February 2016; first published online 3 March 2016

\section{INTRDDUCTION}

Microwave photonics has been an area of research since last decade as it offers several advantages such as wider bandwidth, low loss, high data rate, low weight, immunity against electromagnetic interferences (EMIs), etc. [1, 2]. Although this technology is used in cable network and sensors remoting, its application in radar is still being explored $[3,4]$. Microwave photonics in radar has many applications starting from radio frequency (RF) front end, moving target indication filters to radar signal processing [5]. However, distribution of RF, and digital signals in active electronically scanned array (AESA) radars using microwave photonic network has not been proposed yet.

Photonic application is also found in analog beam-forming network of phased array radars. True time-delay circuits were formed with the help of fiber Bragg grating to synthesize multiple squint-free beams with high instantaneous bandwidth over a wide range of microwave frequencies [6]. An extension of this technology for variable time delay with carrier reference circuit eliminates the need of frequency conversion [7]. In contrast AESA radar uses digital beam forming network

${ }^{1}$ Bharat Electronics Limited, Ghaziabad, Uttar Pradesh, India

${ }^{2}$ Electronics and Communication Engineering Department, ASET, Amity University, Uttar Pradesh, Sector-125, Noida-201313, Uttar Pradesh, India Corresponding author:

M. Mathur

Email: manishamathur@bel.co.in in which digital word is preloaded thus eliminating the need of multiple interconnect functions.

AESA radars are multifunction multibeam radars which scan in azimuth and elevation electronically and thus performs tracking, surveillance, and other functions. The principle of coherent pulse Doppler AESA radar is to preserve the phase of transmitted pulse and compare it with that of receive echo to arrive at target information [8]. The requirement of narrow receive pencil beam increases antenna aperture dimensions, leading to a sizeable number of transmit/ receive $(T / R)$ modules physically spread across the antenna aperture. Each $T / R$ module requires a number of $R F$ and data cables. The layout of antenna array in an AESA radar is a challenge in comparison to conventional reflector based radar antenna.

Conventional scheme for distribution of RF and digital signals are through coaxial and multicore cables. They are inherently lossy and mechanically complex considering weight and volume constraints of multichannel rotary joint. In contrast, optical systems are immune to EMI and electromagnetic compatibility (EMC) issues while providing large bandwidth with increased data rate $[9,10]$. Antenna remoting is possible only because of optical rotary joint as optical cables are almost lossless over long distances [11]. Reliability, maintainability aspect, and RF leakage over a period of time are other drawbacks of conventional coaxial cables. On the other hand, optical system exhibit little field issues [12]. However, the discussion in [12] is focused around photonic sensor feedback system for AESA radar and does not include RF distribution using photonic network. 
Optical system also offers advantage in terms of wavelength division multiplexing (WDM) where a large number of carriers can be transmitted over a single fiber [13]. Use of RF over fiber technology is also presented for ultra accurate optical clock distribution network [14] and RF reference distribution for KEKB particle accelerator [15]. However, its application in radar for RF distribution is still being explored.

Harmonic distortion is an important parameter while adopting photonic systems for AESA radar. Harmonics are generated because of multiple reasons. One of the reason is conversion of receive echo into two level of intermediate frequency (IF) before analog to digital conversion and signal processing. The other reason is frequency agility of radar. Yet another reason for harmonic distortion is nonlinear behavior of optical fiber and generation of intermodulation products in WDM. So, a detailed analysis of harmonic distortion is necessary to validate the proper operation of AESA radar.

All the T/R modules are individually calibrated in near field test range to define digital value of phase and amplitude weight function in AESA radar. While in case of an optically controlled analog beam former network same is done using chirped fiber grating with the help of variable optical delay lines [16]. However, this leads to increase in multiple interconnects. Commercial availability of ruggedised optical components has made technology available where fully photonic based radar is seen as evolution of next generation radars [17]. Integrated microwave photonic systems are another roadmap for optically controlled antenna beams of radars with reconfigurability options [18]. The same integrated microwave photonics concept is extended to variable optical phase shifter to achieve wideband operation upto $18 \mathrm{GHz}$ [19].

Radar operation is carried out over multiple narrow frequency bands to maintain frequency agility. The response of the total chain from input RF followed by RF to optical converter, multiplexer, amplifier, demultiplexer, splitter, and finally regeneration of RF leads to wide variation in the RF signal. This paper proposes to limit the RF output variation within $\pm 1 \mathrm{~dB}$ over full RF band of $2-4 \mathrm{GHz}$ through judicious addition of dynamic control blocks.

The experimental work presented here proposes an application of microwave photonic network to distribute RF and data for AESA radar. Feasibility study of this design has been presented in [20] where phase delay of $800 \mathrm{~ns}$ is observed for $150 \mathrm{~m}$ spool of optical fiber. Detailed power budget calculation and microwave photonic link characterization is presented in [21].

The main contribution of this work is to present a better alternative in the form of microwave photonic network in place of coaxial cable distribution network for AESA radar. The present coaxial cable network imposes a limit on antenna remoting distance to few meters only. Microwave photonic network enables the antenna to be placed kilometers away from operational site as optical cables have a loss of around $0.2 \mathrm{~dB} / \mathrm{km}$ only. This gives tactical advantage for radar operation as it can be controlled from remote. Further the coaxial cable network is prone to EMI/EMC troubles while optical systems are immune to this phenomenon. Also, the overall weight gets reduced to one-fourth compared with a coaxial cable network.

The objective of the design is to limit the variation in RF signal at each digital receiver module within $\pm 1 \mathrm{~dB}$. Each module requires RF signal from a single local oscillator signal. Hence, identical performance of all receivers is also critical for this design. The scope of this work is limited to design of optical distribution network and realization for AESA radar and does not cover antenna radiation characteristics. The design proposed is modular in nature and can be optimized for radars with different range. The paper is organized as follows: Section II presents microwave photonic network for RF distribution in AESA radar. Design guidelines and realization challenges are presented in Sections III and IV, respectively. Section V presents the design of the proposed modules. Experimental results are presented in Section VI. Finally, conclusion is given in Section VII.

\section{MICROWAVEPHOTONIC} NETWDRK

Microwave photonic network for subarray of 64 elements for AESA radar is presented. The design is expandable for more number of elements depending upon radar antenna aperture requirements. The scheme addresses the transmission of four RFs and one digital multiplexed radar signal over single fiber for a distance of about $1000 \mathrm{~m}$. So, scattering losses, relative intensity noise, etc. are not considered in this design. The design also includes module status monitoring and fault reporting system over ethernet considering remote operation of radar. The complete scheme is implemented with the help of three major hardware modules and its associated interconnect cables as shown in Fig. 1. There is a single electrical to optical transmitter and 64 optical to electrical receivers forming the network. Thus, identical transmitted signal is available at each receiver input for processing received echo for pulse Doppler radar measurement.

In this work, coarse WDM is preferred in contrast to dense wavelength division multiplexing (DWDM) for distribution of RF and digital signals over single optical fiber, as with less number of channels, it is simple and cost-effective. Another added advantage of CWDM is that due to $20 \mathrm{~nm}$ channel spacing the effect of harmonic distortion and intermodulation products are reduced. RF over fiber is realized with the help of internal modulation direct detect technique as radar operates in microwave S band [22]. High-efficiency optical receiver alone is not sufficient to compensate the loss caused by splitter. Hence, erbium doped fiber amplifier (EDFA) has been considered in this scheme. The optical channels selected are C and L band due to easy availability of optical amplifiers in this range. Though RF over fiber links with low-noise figure are also reported in [23], but in this work, noise figure of the link is optimized with the help of pre and post RF amplifiers.

Spur free dynamic range (SFDR), minimum discernible signal (MDS), and third-order intercept (TOI) are used for designing and realization of microwave photonic network. Design has been validated by measuring optical power levels, response of system over varied RF input power, measurement of harmonic suppression, noise floor, cable loss, etc. over frequency band of interest. Entire hardware has been designed for use in ruggedised military environment compliant to standard MIL 810F [24].

The modules used in this microwave photonic network are described below:

(A) Electrical to optical converter module (Module 1): The design objective of this module is to tune input RF 




Fig. 1. Proposed microwave the photonic network.

signals to match the optimum signal level required at distributed feedback (DFB) laser. DFB lasers are preferred over VECSEL in 1300-1700 $\mathrm{nm}$ range, as narrow beam width is easier to achieve [25]. It provides a wide dynamic range and a high-power output. All eight channels including digital data are multiplexed with the help of WDM multiplexer to send data on a single-fiber optical cable. Low-power signals from DFB laser are multiplexed; hence, the effect of harmonic distortion is minimal here. This module receives four RF signals at the input of signal conditioning card. All four RF channels in $S$ band are attenuated independently to maintain signal level of $-14 \mathrm{dBm}$ at the input of laser. Test selectable devices are used to tune the circuit at the laboratory level to limit the variation within $\pm 1 \mathrm{~dB}$. The signal conditioning card consists of fixed pi attenuators and variable attenuator for finer adjustments. This attenuated signal is fed to electrical-to-optical converter module, which uses DFB laser to give single optical output at $10 \mathrm{dBm}$ with the help of WDM multiplexer.

This module is fed by $28 \mathrm{~V}$ DC source and total power consumption of this module is found to be $<100 \mathrm{~W}$ measured at input supply. Power supply design provides under voltage, over voltage, and short-circuit protection. It is monitored by a control PCB, which uses Spartan-field programmable gate array (FPGA) and bias power status is monitored and controlled for optical modules. It communicates its own status to central system over ethernet packets.

(B) Optical amplifier and splitter module (Module 2): This module receives optical data from Module 1 , over a fiber link from rotary joint and demultiplexes it into different $\mathrm{C}$ and $\mathrm{L}$ channels and amplifies each channel output. All five light channels are multiplexed again and sent over optical fiber using WDM. The multiplexer multiplexes high-power optical channels; hence harmonic distortion are likely to be generated. Design care is taken to use individual EDFA for each channel so that intermodulation products are reduced. Signal is maintained at $17 \mathrm{dBm}$ with the help of automatic light control feedback system available in EDFA. Optical channels are then split into 64 signals using an array of 1:8 splitters, each channel carrying multiplexed information of eight signals. Loss of the order of $12 \mathrm{~dB}$ in 1:8 splitter is compensated by optical amplifier. These 64 signals are transported to different digital receivers spread across antenna panel of approximately $7 \times 5 \mathrm{~m}^{2}$ size. The basic principle of pulse Doppler radar is based on phase difference measurement between transmitted pulse and received echo. This calls for duplicating 64 optical to electrical receiver spread over the antenna array. The major design challenge is to keep the variation among these 64 group receivers within $\pm 1 \mathrm{~dB}$.

There is a control card in the module which monitors the status, amplification and bias power of optical amplifier. It also monitors its own status and communicates with central unit over RS422 interface. Power supply board provides the required power to each module and its consumption is $<150 \mathrm{~W}$.

(C) Optical to electrical receiver module (Module 3): This module receives the signal from amplifier and splitter module, demultiplexes it using the CWDM demultiplexer and converts back into RF signals using optical to RF converter. The slope efficiency of converter used is of the order of $0.85 \mathrm{~A} / \mathrm{W}$. This gives electrical output of the order of $-25 \mathrm{dBm}$. These RF signals are amplified and processed in electrical domain using signal conditioning cards to match the power levels of digital receivers. Test selectable devices have been used to tune the circuit 
and special purpose low-noise amplifiers followed by low-pass filters to limit the variation in the microwave signal output within $\pm 1 \mathrm{~dB}$.

Its control card monitors the status of optical modules and sends information to central station over $\mathrm{RS}_{422}$ interface. Power supply provides the required power to each module with each receiver consuming $<500 \mathrm{~W}$ of power from a source of $28 \mathrm{~V}$ DC.

\section{DESIGN GUIDELINES}

Conventional design cycle is followed to design and realize microwave photonic network. It starts with review of design input, subsystem design, component selection, design review at various stages, design verification, validation, integration, and testing. Some of the major design criteria are listed below:

(A) Power budget calculation: This is fundamental step in design to finalize individual components. The power carrying capacity of rotary joint, receiver, waveform generator is required to be matched with the available commercial hardware [21].

(B) Optical rotary joint: Antenna remoting requires optical rotary joint to carry multiplexed signal from baseband circuitry to rotating antenna array. This requires power capacity calculation of optical channel of rotary joint.

(C) Component selection: To meet the requirement of harsh military environment, all individual components, connectors, cables, etc. are chosen carefully to meet the operational temperature range as specified -20 to $+55^{\circ} \mathrm{C}$.

(D) Distortion: Non-linear effects of distortion are minimized with the use of proper shielding and grounding. Optical devices are used in linear region to avoid distortion due to gain compression. As WDM introduces intermodulation products, CWDM is preferred over DWDM.

(E) EDFA: EDFA properties allow only limited band operation. Hence, receive data are demultiplexed and amplified individually to have flat gain response. The optical channels are multiplexed again after the EDFA operation. This also helps in reducing harmonic distortion and intermodulation products.

\section{REALIZATION CHALLENGES}

Following are some of the major realization and maintenance aspects of microwave photonic network for AESA radar.

(A) Mechanical design: The fitment of receiver modules is spread out over the antenna array. As the T/R modules and other electronic circuitry generate heat, the mechanical housing design is optimized for air-cooled operation in harsh military environment.

(B) Shock and vibration: The radar will be continuously rotating in azimuth to maintain volumetric coverage for the surveillance functions. All the modules fitted in the antenna array also rotate with the antenna. This requires ruggedization of the modules to withstand shock and vibration, induced due to rotation.

(C) RF and optical cable assembly: The optical system is highly sensitive to dust particles. All the assembly is completed in a clean room rated to $1000 \mathrm{ppm}$ dust level. Around $4 \mathrm{~dB}$ variations have been observed in output power by repeated experimental measurement due to dust accumulation. This variation is controlled with the help of dust measurement on connector surfaces and cleaning with the help of tool JDSU P 5005 i before testing.

(D) Health monitoring and control: Since the modules are located at different physical locations, maintenance in field is a challenge. Further, radar requires continuous operation. Hence, dynamic health update is a mandatory requirement. A separate graphical user interface (GUI) is developed with the help of control card to check health status and control. This can be viewed at the remote location with the help of Ethernet connection.

(E) Safety: Considering laser hazards for eyes, all highlevel optical signals are kept under sealed mechanical housing to protect damage to the operator.

\section{DESIGN DF PRDPDSED MロDULES}

Microwave photonic network described in Section II has been implemented following the design guidelines described in Section III. Photonic hardware modules, power supply boards, control card and associated cables along with mechanical layout design has been realized to meet the required specifications considering the realization challenges described in Section IV. Multichannel EDFA is optimized for C and L band to give constant light output at $17 \mathrm{dBm}$ irrespective of input optical power. Slope efficiency and modulation depth is also selected for optimum performance by appropriate selection of DFB laser and photo detector. Further a $10 \mathrm{~dB}$ gain is added in receivers at the RF level mainly to isolate the photodiode from the output connector as photodiodes are very sensitive to electrostatic discharge and power surges. Adding this gain stage increases the field reliability of receivers. These are some of the design features added to increase the performance limits as explained in [26]. Online calibration feature is also added with the help of FPGA for maintenance purposes.

Following critical parameters are utilized for the design of the proposed microwave photonic network.

(A) Optical power of laser: It is obtained by turning ON the laser with DC current. RF modulation is added to the laser's DC bias current using a bias-tee and direct modulation method [27]. Mean optical power of the carrier output by the laser depends on the bias current. Increasing or decreasing input RF power level does not change the average optical power of the carrier output but changes the modulation index of the optical carrier. The laser's optimum input RF power is governed by $1 \mathrm{~dB}$ compression point and TOI point. In a linear region increase in input power level by $1 \mathrm{~dB}$ increases output power level also by $1 \mathrm{~dB}$. As the unit goes into saturation, it becomes non-linear, resulting in clipping of the waveform. This non-linearity is measured with a TOI measurement. The TOI is a common figure of merit for the intermodulation distortion produced by the amplifier. This TOI point is never attained in practice due to amplifiers gain compression effect. With fiber optic links, TOI point is considered $8-10 \mathrm{~dB}$ above $1 \mathrm{~dB}$ compression point. As a thumb rule, most system designers consider operating the component at $10 \mathrm{~dB}$ below the $1 \mathrm{~dB}$ compression point as a good practice [28]. 
(B) Modulation index: It is a measure of the depth by which a carrier is modulated, expressed in percentage. At low bias currents, the laser will turn OFF. The design value of modulation index must be appropriate, so that neither laser is turned OFF nor compresses power. DFB laser has an operational limitation of $10 \%$ modulation index due to relaxation resonance frequency limit in the linear region and hence the same has been used in this work.

(C) SFDR: It is the range from MDS on the lower side to power compression on the higher side and is given by (1). Noise floor is the theoretical minimum noise for a given bandwidth and temperature. Hence, for a $1 \mathrm{~Hz}$ bandwidth at room temperature the noise floor is calculated in (2).

$$
S F D R=\frac{2}{3}(T O I-N F-10 \log (k T B)),
$$

where $T O I$ is input third-order intercept of the link in $\mathrm{dB}$, $N F$ is the noise figure of the link in $\mathrm{dB}, k$ is the Boltzman constant, $T$ is the temperature in Kelvin and $B$ is the receiver bandwidth in $\mathrm{Hz}$.

$$
\begin{aligned}
\text { Noise Floor } & =10 \log (k T B) \\
& =10 \log \left(1.38 \times 10^{-23} \times 290 \times 1 \times 1000\right) \\
& =-173.97 \mathrm{dBm}
\end{aligned}
$$

(D) MDS: It is minimum input power or the sensitivity of the fiber optic link and is a function of the receiver filter bandwidth and the noise figure of the link. It is designed for $12 \mathrm{~dB} \mathrm{NF}$ at the frequency of interest using (3). The wider the filter bandwidth at the receiver end, the more thermal noise power will come through it. That in turn would raise the noise floor and make it less sensitive to weak signals.

$$
\begin{aligned}
M D S & =k T B+N F=-173.97+12 \\
& =-161.97 \mathrm{dBm} .
\end{aligned}
$$

This implies that any signal greater than $-161.97 \mathrm{dBm}$ is detectable on a link with $12 \mathrm{~dB}$ NF.

(E) Input TOI: It is typically $8-10 \mathrm{~dB}$ higher than the input $1 \mathrm{~dB}$ compression point. $1 \mathrm{~dB}$ compression point is a measure of components power handling capacity. In this work, the typical value of input $1 \mathrm{~dB}$ compression point is $-14 \mathrm{dBm}$ as per manufacturer's datasheet, so input TOI is considered at $-4 \mathrm{dBm}$. SFDR is calculated using (1) and is given in (4).

$$
S F D R=\frac{2}{3}(-4-12+174)=105.33 \mathrm{dBm} .
$$

All active devices exhibit non-linearity which results in harmonics and intermodulation products, which may fall within the bandwidth of interest and are hard to filter out because of the proximity to the carriers. Also they grow in power three times faster as $1 \mathrm{~dB}$ increase in carrier level causes the third-order products to increase by $3 \mathrm{~dB}$. This system is designed at SFDR of approximately $106 \mathrm{dBm}$, which implies that with MDS at $-161 \mathrm{dBm}$ of the input signal can be well tolerated up to $-55 \mathrm{dBm}$ without degraded performance.

(F) Optical power (average) of photodiode: It depends on slope efficiency of photodiode. Slope efficiency represents the transfer function of a laser diode called responsivity. Optical power figure does not play a large role in the fiber optic link performance. For $10 \mathrm{~mW}$ optical carrier with $10 \%$ modulation index, output power swings between 9 to $11 \mathrm{~mW}$. If the responsivity of photo diode is $0.85 \mathrm{~A} / \mathrm{W}$, e.g. the peak to peak current will be $0.00085 \mathrm{~A}$. Power output for root-mean-square value of current at matched load of $50 \mathrm{ohm}$ will be $-17 \mathrm{dBm}$. The receiver has about $17 \mathrm{~dB}$ RF gain after the photodiode. Thus, the link is zero $\mathrm{dB}$ link with pre and post RF amplification.

\section{EXPERIMENTAL SETUP AND RESULTS}

Figure 2 shows block diagram of experimental setup and Fig. 3 shows actual photograph of the setup. All optical connectors are cleaned and inspected with optical inspection instrument JDSU P 5005 i before connection. RF cables are calibrated for all spot frequencies of radar with o $\mathrm{dBm}$ input power. Testing was carried out in $2-4 \mathrm{GHz}$ band. The link is designed to keep the RF output variation within $0 \pm 1 \mathrm{~dB}$ for full $\mathrm{RF}$ band of 2-4 GHz. Optical power is measured at output of transmitter with optical spectrum analyzer at different wavelengths for all 64 channels. The frequency response of photo diodes is measured with Agilent Spectrum Analyzer PSA E4445A with resolution and video bandwidth of $910 \mathrm{~Hz}$, for all 64 receivers. The measurement at spectrum analyzer was possible with the help of RF gain block circuit present in each receiver module. These values are used to tune the signal conditioning cards at each receiver for specific RF band. The analysis shows that a separate signal conditioning card is required at transmitter and receiver level to equalize the performance for full RF band.

Variation in received RF power level is observed for all 64 channels due to cascading effect of splitter performance. This restricts interchangeability of receiver modules. The uniformity in the result is possible with the use of 1:64 splitter where channel to channel separation is uniform and fixed. Further a variation of $4 \mathrm{~dB}$ is also observed in electrical to optical converter. This variation is compensated with the help of RF signal conditioning cards.

The optical transceivers are designed to operate in linear region only and the output depends on responsivity of photodiode. Figure 4 shows response of optical transceiver for input RF power variation. It can be observed from Fig. 4 that output power level is varying linearly with respect to input power level till $24 \mathrm{dBm}$, i.e. $10 \mathrm{~dB}$ below $1 \mathrm{~dB}$ compression point.

Microwave photonic link has been analyzed by measuring harmonic distortions to validate proper operation of radar.

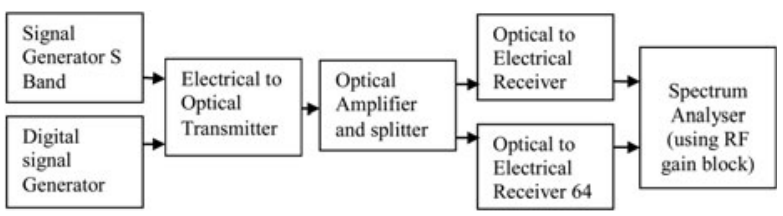

Fig. 2. Block diagram of the experimental setup. 


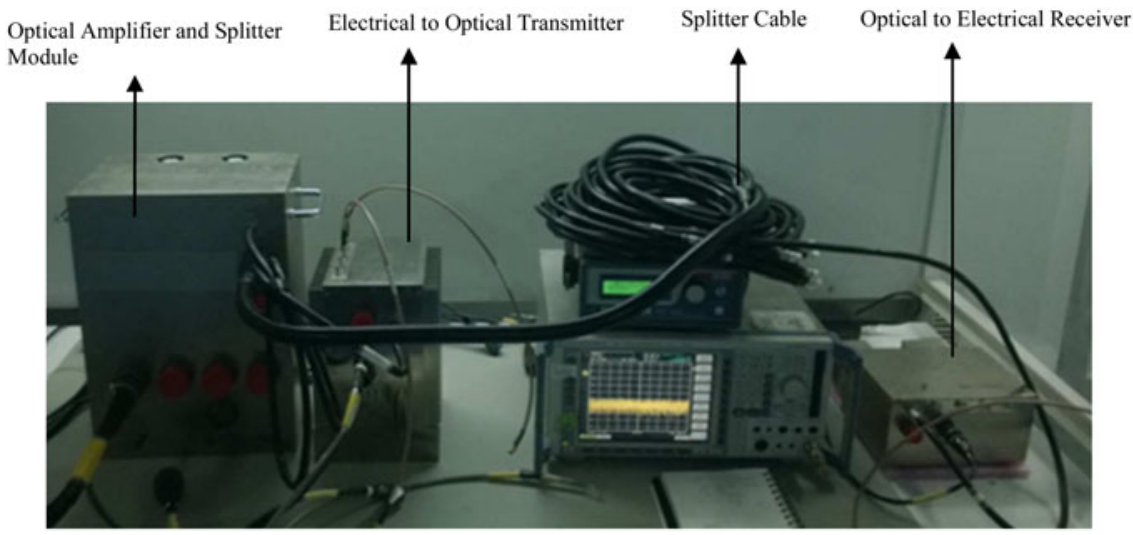

Fig. 3. Actual photograph of the experimental setup.

TOI is an extrapolated point which depends on third-order products and may affect frequency of interest. Harmonic distortion has been measured over full frequency band of 2${ }_{4} \mathrm{GHz}$ with a small step size of $100 \mathrm{MHz}$ to analyze this effect. As third-order products are close to carrier, the measurement of harmonic distortion has been carried out upto the fourth level considering that fifth harmonic is small in amplitude [29]. Further the mixers used in receiver for down conversion of frequency are followed by band pass filters; hence, frequencies of the order of fifth and above harmonics, will be rejected by band pass filters. Table 1 shows second, third, and fourth-order components of harmonic distortion. As a design thumb rule, harmonic suppression four times below fundamental signal is considered acceptable. For $-14 \mathrm{dBm}$ input RF signal, the second, third, and fourth harmonic should be four times below carrier, i.e. $56 \mathrm{dBc}$. It can be observed from Table 1 that second, third, and fourth harmonic distortion is $<62 \mathrm{dBc}$. So, it meets the specification of $56 \mathrm{dBc}$ for all spot frequencies of radar.

Noise floor has been measured using spectrum analyzer for 2-4 GHz RF band. RG142 RF SMA to SMA cables has been used. Using (2) average noise floor computed for a bandwidth of $2 \mathrm{GHz}$ is $-81 \mathrm{dBm}$. It can be observed from the results presented in Table 2 that it meets the specification required for bandwidth of $2 \mathrm{GHz}$.

It can be observed form Tables 1 and 2 that harmonic distortion levels are prominent over noise. Each application of RF over fiber requires a specified harmonic level for satisfactory performance [30]. Radar's dynamic range depends upon

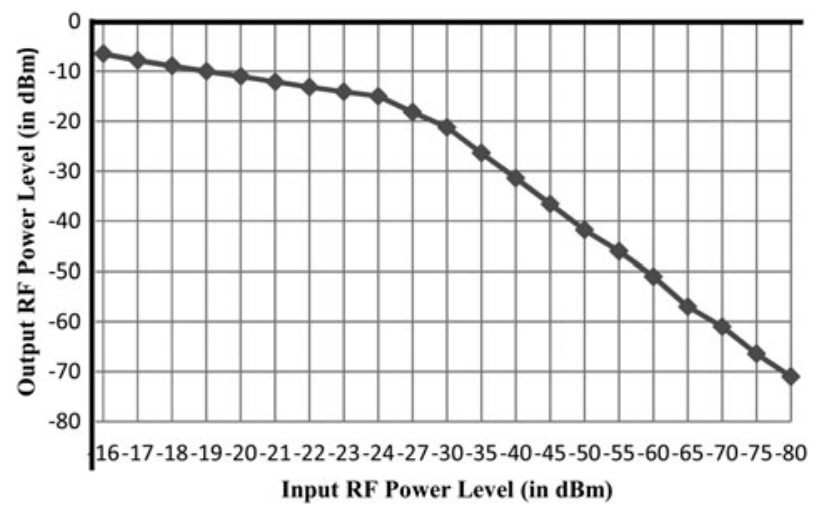

Fig. 4. RF response of the optical transceiver. receiver's noise and harmonic level. In this work, the transmitter's RF signals are transported over optical fiber; hence degradation in harmonic level does not affect the overall performance of radar, but poses an additional constraint in the form of the reduced operational level available for the transmitter. For radar's proper operation, the harmonic level should be well below the noise level, so that its effect is negligible. This condition is acceptable for transmission path. Same application in receive path will limit the system dynamic range for receive echo.

The link budget is validated against calculations presented in [21]. The requirement of same frequency to be split for all digital receivers necessitates for 1:64 splitter, which introduces a loss of $17-20 \mathrm{~dB}$ in the system. To compensate for this loss, EDFA is used which is based on automatic light control logic thus keeping PIN diode input constant over varied frequency range. Figure 5 shows the frequency response of the optical distribution network for complete RF band of $2-4 \mathrm{GHz}$ for o $\mathrm{dBm}$ input RF signal. It can be observed that the variation in output RF power is from -0.4 to $+0.7 \mathrm{~dB}$. So, it meets the design objective to limit the variation within $\pm 1 \mathrm{~dB}$.

Table 1. Harmonic distortion of the second, third and fourth orders.

\begin{tabular}{|c|c|c|c|}
\hline $\begin{array}{l}\text { Frequency } \\
(\mathrm{GHz})\end{array}$ & $\begin{array}{l}\text { Second } \\
\text { harmonic }(\mathrm{dBc})\end{array}$ & $\begin{array}{l}\text { Third } \\
\text { harmonic }(\mathrm{dBc})\end{array}$ & $\begin{array}{l}\text { Fourth } \\
\text { harmonic }(\mathrm{dBc})\end{array}$ \\
\hline 2 & -68.82 & -68.23 & -66.23 \\
\hline 2.1 & -69.72 & -69.32 & -65.23 \\
\hline 2.2 & -70.56 & -68.21 & -67.23 \\
\hline 2.3 & -69.74 & -69.32 & -66.78 \\
\hline 2.4 & -68.56 & -70.23 & -67.1 \\
\hline 2.5 & -69.24 & -69.56 & -66.77 \\
\hline 2.6 & -70.81 & -69.85 & -66.86 \\
\hline 2.7 & -68.74 & -67.78 & -67.23 \\
\hline 2.8 & -70.25 & -67.02 & -65.46 \\
\hline 2.9 & -69.56 & -66.5 & -65.25 \\
\hline 3.1 & -68.84 & -66.37 & -64.42 \\
\hline 3.2 & -69.7 & -67.38 & -65.11 \\
\hline $3 \cdot 3$ & -70.32 & -67.99 & -65.42 \\
\hline 3.4 & -67.59 & -67.48 & -62.86 \\
\hline 3.5 & -67.64 & -68.11 & -63.23 \\
\hline 3.6 & -70.61 & -66.57 & -64.25 \\
\hline 3.7 & -70.98 & -67.37 & -63.98 \\
\hline 3.8 & -71.17 & -67.02 & -63.54 \\
\hline 3.9 & -70.34 & -66.5 & -62.63 \\
\hline 4 & -69.7 & -67.23 & -63.23 \\
\hline
\end{tabular}


Table 2. Noise floor.

\begin{tabular}{ll}
\hline Frequency $(\mathrm{GHz})$ & Noise floor $(\mathbf{d B m})$ \\
\hline 2 & -82 \\
2.5 & -81 \\
3 & -80.5 \\
3.5 & -81 \\
4 & -81.5 \\
\hline
\end{tabular}

Experimental results in this work have met the specification requirement of digital receivers of the antenna array. The RF signals are verified for faithful reproduction at receiver. It justifies the advantage of microwave photonic network for AESA radar. The production hardware modules are realized to be used with existing radar for field performance.

\section{CONCLUSIONSAND DISCUSSION}

Microwave photonic network for RF signal distribution in AESA radar has been designed and validated in this work. The design challenge has been to limit the variation in output RF power to $\pm 1 \mathrm{~dB}$ for multiple spot frequencies over the RF band. Since the behavior of RF varies widely over full band, it has been proposed to use different RF signal conditioning cards both at input and output of RF channel with tunable option to limit the variation. Thus, link has been designed to maintain zero $\mathrm{dB}$ gain with the help of constant gain EDFA. Proposed microwave photonic network for RF distribution over fiber is modular in nature. This suits the application in AESA radar as AESA radar is also modular with the number of $T / R$ modules depending on radar range. The number of receive modules may be increased to meet the requirements of long-range radars. The proposed microwave photonic network offers an additional advantage of antenna remoting with the help of optical rotary joint. This protects the radar operator from harmful high-power RF radiations. Also it protects the operator and equipments during war time as radar is targetted by sensing its RF radiation. Radar performance is highly dependent on the acceptable levels of non-linear distortion. The experimental results including that of harmonic distortion validates the feasibility of the proposed microwave photonic distribution network for AESA radars offering

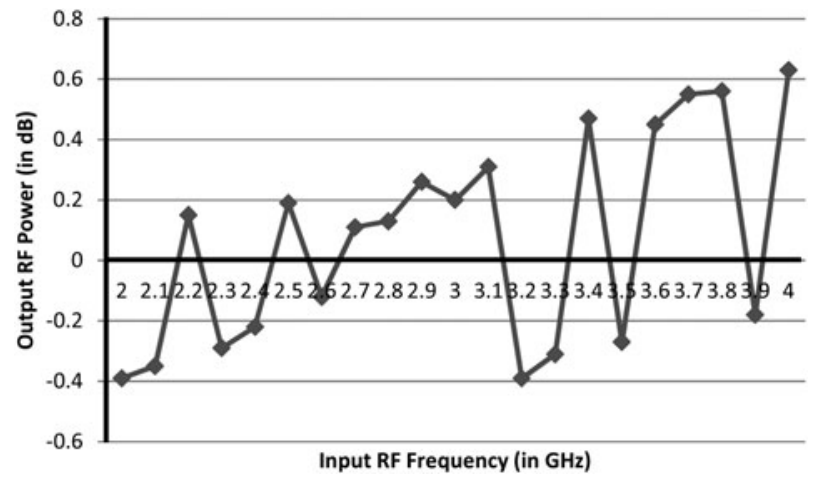

Fig. 5. Frequency response of the microwave photonic network. advantages such as reduced weight, volume, complexity and enhanced data rate, bandwidth, and immunity to EMI and EMC. Further work will be carried out to evaluate the field performance. Future work will be carried out to make the photonic network duplex to reduce the number of cables in the receive path.

\section{REFERENCES}

[1] Seed, A.J.: Microwave photonics. IEEE Trans. Microw. Theory Tech., 50 (3) (2002), 877-887.

[2] Seed, A.J.: Microwave photonics. J. Lightwave Technol., 21 (12) (2003), 3104-3115.

[3] Capmany, J.; Novak, D.: Microwave photonic combines two worlds. Nat. Photonics, 1 (2007), 319-330.

[4] Pappert, S.A.; Krantz, B.: RF photonic for radar front ends, in Proc. of IEEE Radar Conf., Boston, 2007, 965-970.

[5] Pan, S.; Zue, D.; Zhang, F.: Microwave photonics for modern radar systems. Trans. Nanjing Univ. Aeron. Astron., 31 (3) (2015), 219-240.

[6] Minasian, R.A.; Alameh, K.E.: Optical-fiber grating-based beamforming network for microwave phased arrays. IEEE Trans. Microw. Theory Tech., 45 (8) (1997), 1513-1518.

[7] Berceli, T.; Herczfeld, P.R.; Daryoush, A.S.; Rosen, A.: Performance optimization of optically controlled phased array antenna T/R modules, in 19th European Microwave Conf., London, 1989, 945-950.

[8] Skolnik, M.I. (ed.) : Phased array radar antenna, in Radar Handbook, 2nd ed., McGraw-Hill, New York, 1990, 7.1-7.36.

[9] Berceli, T.; Herczfeld, P.: Microwave photonics - a historical perspective. IEEE Trans. Microw. Theory Tech., 58 (11) (2010), 2992-3000.

[10] Gg Yao, J.: A tutorial on microwave photonics. IEEE Photonic Soc. Newslett., 26 (3) (2012), 5-12.

[11] Jager, D.: Microwave photonic-past, present and future. IEEE Microw. Mag., 10 (4) (2009), 154-156.

[12] Vinetti, P.; D’ Urso, M., Dispenza, M.: Increasing phased arrays resilience via photonic sensor network feedback. IEEE Antennas Wireless Propag. Lett., 11 (2012), 201-204.

[13] Mukharjee, B.: WDM optical communication networks: progress and challenges. IEEE J. Sel. Areas Commun., 18 (10), (2000), 1810-1824.

[14] Hsu, M.T.L.; Shaddock, D.A.; Warrington, R.B.; Gray, M.B.: All digital radio frequency signal distribution via optical fibers. IEEE Photonics Technol. Lett., 24 (12) (2012), 1015-1017.

[15] Naito, T.; Ebihara, K.; Suetake, M.; Ezura, E.; Tsukuba, K.E.K.: RF reference distribution using fiber optic links for KEKB accelerator, in Proc. of the IEEE Particle Accelerator Conf., Chicago, 2001, 791-793.

[16] Ortega, B.; Cruz, J.L.; Capmany, J.; Andres, M.V.; Pastor, D.: Variable delay line for phased-array antenna based on a chirped fiber grating. IEEE Trans. Microw. Theory Tech., 48 (8) (2000), 1352-1360.

[17] Ghelfi, P. et al.: A fully photonic based coherent radar system. Nat. Lett., 507 (2014), 341-345.

[18] Burla, M. et al.: Multiwavelength-integrated optical beamformer based on wavelength division multiplexing for 2 -D phased array antennas. J. Lightwave Technol., 32 (20) (2014), 3509-3520. 
[19] Xiaoke, Yi; Huang, T.X.H.; Minasian, R.A.: Photonic beamforming based on programmable phase shifters with amplitude and phase control. Photonics Technol. Lett., 23 (18) (2011), 1286-1288.

[20] Mathur, M.; Sridhar, N.; Rai, J.K.: Design and analysis of RF signal distribution over optical fiber for active aperture radar, in Proc. of the IEEE Int. Conf. on signal processing and integrated networks, Amity University, Noida, India, 2014, 354-357.

[21] Mathur, M.; Sridhar, N.; Rai, J.K.: Microwave photonic link characterization for phased array radar, in Proc. of the IEEE Int. Conf. Australian Microwave Symp. Melbourne, 26-27th June, Melbourne, Australia, 2014, 3-4.

[22] Cox, C. III; Ackerman, E.; Helkey, R.; Betts, G.E.: Techniques and performance of intensity-modulation direct-detection analog optical links. IEEE Trans. Microw. Theory Tech., 45 (8) (1997), 1375-1383.

[23] Ackerman, E.I. et al.: RF-over-fiber links with very low noise figure. J. Lightwave Technol., 26 (15) (2008), 2441-2448.

[24] Department of Defence, USA.; Test method standard for environmental engineering considerations and laboratory tests, 2000.

[25] Xiaodan, P.; Alexander, L.; Vegas Olmos, J.J.; Marta, B.; Roberto, L.: Performance evaluation for DFB and VCSEL based $60 \mathrm{GHz}$ radio over fiber system, in 17th Int. Conf. on Optical Network Design and Modelling, Brest, France, 2013.

[26] Cox, C.H. III; Ackerman, E.I.; Betts, G.E.; Prince, J.L.: Limits on the performance of RF-over-fiber links and their impact on device design. IEEE Trans. Microw. Theory Tech., 54 (2) (2006), 906-920.

[27] Illing, L.; Kennel, M.B.: Shaping current waveforms for direct modulation of semiconductor lasers. IEEE J. Quantum Electron., 40 (5) (2004), 445-452.

[28] Zhu, Z.; Zhao, S.; Tan, Q.; Jiang, W.; Li, Y.; Li, X.: A linearized optical single-sideband modulation analog microwave photonic link using dual parallel onterferometers. IEEE Photonics J., 5 (5) (2013), 5501712-5501712.

[29] Lin, W. et al.: $10 \mathrm{~m} / 500 M b p s$ WDM visible light communication systems. Opt. Express 20 (9) (2012), 9919-9924.

[30] Roselli, L. et al.: Analog laser predistortion for multiservice radio-over-fiber Systems. J. Lightw. Technol., 21 (5) (2003), 12111223.

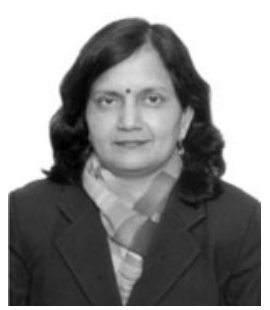

Manisha Mathur received her B.E. degree in Electronics and Communication Engineering from M.I.T.S. Gwalior, India (1990), M. Tech. degree in Digital Communication from M.A.N.I.T. Bhopal, India (1992). Since 1992 she has been working as D.G.M. in Bharat Electronics Limited, Ghaziabad, India. Currently, she leads a team for design and development of active aperture-phased array radar. Her area of interest includes radar signal processing, radar system design, and photonic systems.

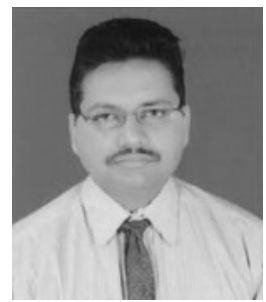

Jaynendra Kumar Rai received his B.E. degree in Electronics Engineering from Shri Guru Gobind Singh College of Engineering and Technology, Nanded, India (1996); M. Tech. degree in Electronics Engineering from Visvesvaraya Regional College of Engineering, Nagpur, India in 1999; and Doctoral degree from Motilal Nehru National Institute of Technology, Deemed University Allahabad, Uttar Pradesh, India in 2011. Presently, he is working as a Professor at the Department of Electronics and Communication Engineering, Amity School of Engineering and Technology, Amity University, Uttar Pradesh, Noida, India. He has published various papers in peer-reviewed international journals and conferences. His current research interests include robotics, medical image and signal processing, and communication engineering.

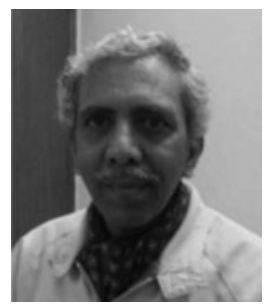

Nilakantan Sridhar completed his M.Sc. (in Phy-Electronics) in 1977, M. Phil (in Phy- Microwaves) in 1979, and Ph.D. in Microwave Antenna field in 1983, from the Department of Physics and Astrophysics, University of Delhi. He was involved in the design and realization of many fixed beam, scanning beam antennas for LOS and Satcom applications, Tactical Deployable antennas, Linear and Planar Phased Array antennas for radar applications, etc. Presently, he is working as Addl. General Manager of Qual. Sys. supporting all the manufacturing divisions of Bharat Electronics Ltd., Ghaziabad Unit. He has a number of publications in peerreviewed international journals and conferences. His current research interests include Microwave Multi beam Antennas, and Active Phased Array Antenna implementations. 